\section{Classification of dopa-responsive dystonia - a patient's perspective}

\author{
Lucy Mottet
}

In a recent Review article (Dopa-responsive dystonia - clinical and genetic heterogeneity. Nat. Rev. Neurol. 11, 414-424; 2015) ${ }^{1}$, Wijemanne and Jankovic proposed that the differential diagnosis of dopa-responsive dystonia (DRD) can be narrowed by defining three clinical presentations: classic DRD, DRD with parkinsonism, and early-onset atypical DRD. As a non-medical academic with genetically confirmed autosomal dominant DRD that was misdiagnosed for nearly 10 years, I support the authors' methodical approach to differential diagnosis. However, owing to my own presentation of DRD, I propose a fourth clinical presentation: atypical late-onset DRD with Parkinson disease (PD).

My own atypical phenotype - adult-onset and rapidly generalizing DRD with $\mathrm{PD}$ - has not, to my knowledge, been described in the literature. My illness had a similar age of onset (41 years) and similar characteristics to the progressive neurological disease that my paternal grandmother died from at 53 years of age. My father had late-onset parkinsonism, and an aunt has late-onset vocal and unilateral hand tremors. Prior to successful bilateral deep brain stimulation (DBS), I had motor fluctuations and crippling biphasic levodopainduced dyskinesia.

My phenotype differs from the three proposed in the recent literature ${ }^{2-6}$, which roughly correspond to those proposed by Wijemanne and Jankovic ${ }^{1}$ : a classic nondegenerative, benign form of dystonia that responds to low, sustained doses of levodopa; a degenerative form (late-onset and without dystonia) with levodopa-induced dyskinesia, parkinsonism and abnormal dopaminergic imaging; and an intermediate form. I believe that the adoption of the term 'atypical', as suggested by Wijemanne and Jankovic, would be valuable in clinical practice.

Several recently published articles discuss the clinical and genetic heterogeneity of DRD phenotypes in relation to the genetics of PD and parkinsonism ${ }^{2-9}$, with implications for the clinical presentations of DRD proposed by Wijemanne and Jankovic. I have the p.K224R mutation in $\mathrm{GCH} 1$, the same genotype as that of some unrelated individuals with PD but not $\mathrm{DRD}^{2,4}$. Segawa et al. ${ }^{10}$ have proposed that the location of a mutation in $\mathrm{GCH} 1$ influences the phenotype of DRD, so the suggestion that some GCH1 mutations cause PD could have considerable implications for people who carry a $\mathrm{GCH} 1$ mutation but do not develop DRD in childhood. Mencacci et al. ${ }^{2}$ suggest that administration of levodopa to such individuals in childhood might protect against later onset of $\mathrm{PD}$, and they point to animal models in which "levodopa has been shown to promote recovery of nigrostriatal denervation." However, an intermediate phenotype was observed in a patient who had been treated with levodopa since childhood: he had abnormal dopaminergic imaging, but no PD symptoms or dyskinesia ${ }^{5}$. This phenotype could indicate that dopamine replacement is not neuroprotective, and that the patient had preclinical $\mathrm{PD}^{3,5}$, but could similarly indicate that dopamine replacement suppressed the emergence of PD.

Recognition among clinicians that $\mathrm{GCH} 1$ mutations can manifest with a range of phenotypes, including adult-onset generalized dystonia and PD, is vital. Use of the word 'atypical' by Wijemanne and Jankovic ${ }^{1}$ in their classification recognizes that the classic paediatric DRD phenotype is not the only one, and recognition of the range of phenotypes can transform the lives of patients.

Lucy Mottet is at the University of Western Australia, 35 Stirling Highway, Crawley WA 6009, Australia. lucy.mottet@yahoo.com

doi: 10.1038/nrneurol.2016.85 Published online 17 Jun 2016

1. Wijemanne, S. \& Jankovic, J. Dopa-responsive dystonia - clinical and genetic heterogeneity. Nat. Rev. Neurol. 11, 414-424 (2015).

2. Mencacci, N. E. et al. Parkinson's disease in GTP cyclohydrolase 1 mutation carriers. Brain 137. 2480-2492 (2014).

3. Mencacci, N. E. et al. Reply: Parkinson's disease in GTP cyclohydrolase 1 mutation carriers. Brain 138 , e352 (2015).

4. Guella, I. et al. Parkinson's disease in GTP cyclohydrolase 1 mutation carriers. Brain 138, e349 (2015).

5. Teerbeek, J., Hermans, S., Laere, K. V. \& Vandenberghe, W. Parkinson's disease in GTP cyclohydrolase 1 mutation carriers. Brain 138, e350 (2015).

6. Lewthwaite, A. J. et al. Novel GCH1 variant in Dopa-responsive dystonia and Parkinson's disease. Parkinsonism Relat. Disord. 21, 394-397 (2015).

7. Carrechio, M. \& Schneider, S. Hot topics: GTP cyclohydrolase 1 mutations and Parkinson's disease: new insights beyond dopa responsive dystonia. Mov. Disord. 39, 910 (2015)

8. Furukawa, Y. $\&$ Kish, S. J. Parkinsonism in GTP cyclohydrolase 1 deficient DOPA-responsive dystonia. Brain 138, e351 (2015).

9. Ryan, B. J., Crabtree, M. J., Channon, K. M. \& Wade-Martins, R. Parkinson's disease in GTP cyclohydrolase 1 mutation carriers. Brain 138, e348 (2015).

10. Segawa, M., Nomura, Y., Yukishita, S., Nishiyama, N. $\&$ Yokochi, M. Is phenotypic variation of hereditary progressive dystonia with marked diurnal fluctuation/ dopa-responsive dystonia (HPD/DRD) caused by the difference of the locus of mutation on the GTP cyclohydrolase $1(\mathrm{GCH}-1)$ gene? Adv. Neurol. 94, 217-223 (2004)

Competing interests statement

The author declares no competing interests 